\title{
Why-Type Question to Query Reformulation for Efficient Document Retrieval
}

\author{
Manvi Breja, National Institute of Technology, Kurukshetra, India \\ (iD) https://orcid.org/0000-0003-0607-3094 \\ Sanjay Kumar Jain, National Institute of Technology, Kurukshetra, India \\ D https://orcid.org/0000-0003-1999-5530
}

\begin{abstract}
Understanding the actual need of users from a question is very crucial in non-factoid why-question answering as why-questions are complex and involve ambiguity and redundancy in their understanding. The precise requirement is to determine the focus of question and reformulate them accordingly to retrieve expected answers to a question. The paper analyzes different types of why-questions and proposes an algorithm for each class to determine the focus and reformulate it into a query by appending focal terms and cue phrase 'because' with it. Further, a user interface is implemented which asks input why-question, applies different components of question, reformulates it, and finally, retrieves web pages by posing query to Google search engine. To measure the accuracy of the process, user feedback is taken which asks them to assign scoring from 1 to 10 , on how relevant are the retrieved web pages according to their understanding. The results depict that maximum precision of $89 \%$ is achieved in informational type why-questions and minimum of $48 \%$ in opinionated type why-questions.
\end{abstract}

\section{KEYWORDS}

Constituency Parsing, Dependency Parsing, Document Retrieval, Non-Factoid, Precision, Question Answering System, Question Reformulation, User Feedback

\section{INTRODUCTION}

Question Reformulation is one of the components of Question Analysis module in Question Answering System. Question Reformulation reformulates the input question according to user's need in order to affect the accuracy of subsequent modules. Why-type non-factoid questions are complex and ambiguous; making them difficult to answer. It is difficult to understand the actual need of user and derive an appropriate non-ambiguous meaning to it. If a correct query is posed to a search engine, it retrieves appropriate web pages that ultimately help in accurate document retrieval. In English language, there are two broad categorizations of questions (1) Factoid questions of type what, where, which, when and who; (2) Non-Factoid questions of type why and how. The factoid questions are simple and non-ambiguous whereas non-factoid questions are complex and difficult to answer.

Question Reformulation plays a crucial role in question answering system. It retransforms question into an appropriate query that depicts the user's need and thus helps in efficient answer retrieval. The performance of question reformulation affects the performance of subsequent modules, i.e. document, answer candidate extraction and answer re-ranker (Kangavari et al., 2008). 
Query reformulation is a key task in today's web search engines for retrieving accurate and best results corresponding to the users' query. Query reformulation is a process of modifying original query to resolve problems of ambiguity, vocabulary mismatch and vagueness. There are different techniques to query reformulation viz. (1) query expansion, (2) query suggestion and (3) query refinement (Ooi et al., 2015).

Query expansion expands query based on (a) relevance feedback by finding co-occurring terms, (b) query terms appended by their synonyms retrieved from WordNet and (c) retrieved informative terms for expansion from definition clusters (Bernhard, 2010). Query refinement modifies query based on the users' past query logs. It doesn't provide choice to user in selecting terms which can be appended to query. Terms are generated based on user feedback from the top ranked documents irrelevant to its appropriateness which helps in achieving high recall and precision. Finally query suggestion helps understanding the actual information need of user and is found as the most fundamental features of search engines. They are often required in case of rare query being posed, single-term query, unambiguous query suggestions, query suggestions are generalized form of original query and several pages are crawled by user. The approach suggests several other refined query corresponding to original user query based on the users' interest/search logs analysis so that user can select terms that should be replaced original terms for better document retrieval.

The paper focuses on improving the why-question answering system by reformulating whyquestions into an appropriate query that can depict the user's need and when posed on search engine, help in retrieving appropriate web pages. There are some cases where the actual user need can't be understood from the question, thus there comes the need for analyzing the question and reformulates it into an appropriate form that can depict the user need from the question.

The organization of paper is described as: Section 2 discusses researches of reformulation. Section 3 puts light on the main focus of the article. Section 4 analyzes different components of question with their impact on reformulation. Section 5 discusses algorithm designed for reformulation of different why-type questions. Section 6 highlights implementation details utilized while designing a user interface for reformulation. Section 7 describes results with their analysis on user feedback. Finally section 8 concludes the work.

\section{BACKGROUND}

This section highlights different researches done for improving question answering system utilizing query reformulation and expansion. Kangavari et al. (2008) identified various ways to express answers to a question in question reformulation component. The authors adopted syntactic and semantic relations between words of question, utilizing patterns and other information of previous existing questions which are similar to users' question. Herdagdelen et al. (2010) utilized integrated syntactic and semantic models with Levenshtein distance algorithms for reformulating query which improved the performance in retrieving documents. Pires (2012) developed JustAsk QAS with Query classification and Reformulation to improve passage retrieval by understanding users' information need. They adopted reformulation techniques by designing 13 matching patterns at lexical level. Umamaheswari et al. (2012) utilized semantic based reformulation technique by generating patterns on the basis of lexical, syntactic and semantic constraints. The technique is applied on TREC dataset to retrieve their accurate answer. Each retrieved candidate answer is weighted on the parameters of length, semantic similarity between Question \& Answer and distance between keyword to attain a precision of 0.49. Musa et al. (2019) proposed a QAS architecture comprising three modules (a) Rewriter module which reformulates a question into queries by selecting important terms using ConceptNet embeddings, (b) Retriever module which retrieves relevant passages corresponding to queries and (c) Resolver which utilizes textual entailment probabilities to determine the best final answer. Esposito et al. (2020) proposed hybrid query expansion method which extracts synonyms and hypernyms of question terms from MultiWordNet. The resulting set is ranked based on different question words and senses. 
Relevant document sentences are retrieved and effectiveness is measured $\&$ assessed for candidate answer sentences. Herrara et al. (2021) proposed reformulation of Spanish questions as a component of QAS. Questions are reformulated into new individual questions based on the question elements such as lexical category of each term, named entities and multi-word terms. Further the grammatical elements are identified on different question classes trained on CNN models which further help to locate the question focus in order to rephrase it properly. Vakulenko et al. (2021) discussed question re-writing component for conversational QA. Ambiguous question asked in conversational context are reformulated into unambiguous question. The method was adopted for two tasks i.e. retrieval QA for finding an answer to a question as a ranked list of passages and extractive QA for finding an answer to a question as a text span within a passage. Different Question Reformulation (QR) models are tested where Transformer++ performed best with 0.81 ROUGE score on CANARD dataset and 0.9 ROUGE score on TREC CAsT dataset.

\section{MAIN FOCUS OF THE ARTICLE}

Why-questions are complex and involve variability in their answers depending upon the need of the user. There is a need to accurately understand the users' requirement from question in order to retrieve appropriate documents and answer candidates. The users' need from the question is highly dependent on determining the main question focus which sometimes requires reformulation in case of short redundant questions. Since finding one correct answer to non-factoid why-type questions require extensive analysis of questions which sometimes demands question reformulation which can be interactive or non-interactive. The paper contributes in designing an algorithm for reformulating question into query based on different question types proposed in the research by Breja and Jain $(2017 ; 2018)$. This objective is achieved by carrying out following three steps:

1. Analyzing different components of question with their impact on reformulation.

2. Designing algorithm for reformulating question into query based on different why-type question.

3. Developing a user interface for why-question to query reformulation which outputs features of question, reformulates it and takes user feedback on the retrieved web pages.

\section{DIFFERENT COMPONENTS OF QUESTION AND THEIR ROLE ON REFORMULATION}

There are different characteristics of question which play crucial role in understanding the process of reformulation.

1. Named Entity Recognition: It is one of the subtasks for information extraction from natural language text. It identifies real world entities from the text. In python, there are two ways to identify named entities in a question (1) using Stanford Core NER and NLTK which recognizes three classes of named entities viz. 'Location', 'Person', 'Organization' and 'O' as a background tag which don't fit any of the three labels. (2) using SPACY which supports various types of entities such as 'Person', 'NORP', 'FAC', 'ORG', GPE, 'LOC', 'PRODUCT', EVENT, DATE, TIME, PERCENT, MONEY and many more (Levengood, 2020). The paper utilizes SPACY to identify named entities from the question that reflects the role of each entity in a question.

2. Tokenization: Tokenization is a process of breaking text into smallest unit called tokens. Each why-question is tokenized and separated into tokens using 'word_tokenize' in NLTK.

3. POS Tagging: POS tagging is a process to assign part of speech tag to each tokenized word in a sentence. The paper applies POS tagging using NLTK to assign tags to each token of why-type question. 
4. Lemmatization: Lemmatization is a process of removing inflectional endings of words and output their base or dictionary form, termed as lemma. It is better than stemming as it considers the context of each word to perform morphological analysis on them. In Python, there are 9 approaches to implement lemmatization; (1) WordNet, (2) WordNet + POS tag, (3) TextBlob, (4) TextBlob +POS tag, (5) spaCy, (6) TreeTagger, (7) Pattern, (8) Gensim, and (9) Stanford CoreNLP (Prabhakaran, 2021) . The paper applies spaCy module to lemmatize each word of why-question as it overcomes limitations from other approaches.

5. Sentiment analysis: Sentiment analysis also termed as opinion mining is a process of determining polarity of text, whether it is a document, paragraph, sentence or phrase. Polarity is categorized as positive, negative or neutral which ultimately reflects the opinion, attitude or emotions of speaker/writer. The paper performs sentiment analysis of question using VADER tool. It is an efficient to predict the positivity or negativity of a text with their magnitude.

6. Noun phrase extraction: This helps to determine a list of noun phrases in the question text. It is utilized using noun_phrases property of TextBlob in python.

7. Constituency parsing: Constituency parsing utilizes constituent-based grammar to analyze and extract the constituents of a text which represents its internal structure. It breaks the sentences into its constituents according to phrase structure rules of grammar. These rules help to determine the ordering and hierarchical structure of constituents in sentence. Each user input question is parsed to analyze the syntactic structure of its constituents such as NP (noun phrases), VP (verb phrases) and PP (Prepositional phrases) and many more. It is implemented using StanfordCoreNLP parse method (Bengfort, 2018).

8. Dependency parsing with tree formation: Dependency parsing is another type of parsing which analyzes the grammatical structure of text by considering the dependencies involved between each words in a sentence. It uses dependency-based grammars to analyze the syntactic and semantic dependencies with relationships between tokens of a sentence. Dependency relationship of each user question is constructed using displacy method of spacy and the whole dependency tree is visualized by converting spacy tree to nltk tree (Bengfort, 2018).

\section{ALGORITHM TO REFORMULATE EACH QUESTION TYPE}

This section discusses an algorithm which is proposed to reformulate Why-type questions into an appropriate query. The reformulated query helps in better retrieval of documents if posed on search engines.

There are positive and negative why-type questions. Negativity affects the query reformulation. A taxonomy is proposed for why-type questions by Breja and Jain (2018) which is categorized as (1) Informational which seeks reasoning about the facts, (2) Historical which seeks reason of the events occurred in past, (3) ComparativeSituational which asks reasoning for comparison of events occurred at a particular situation or circumstance, (4) Situational seeks reasoning for events occurred at a particular situation and (5) Opinionated which asks about the opinions on some person or product.

Rules are analysed for each type of why-questions and important focus terms are visualized which play a significant role to formulate query posed on search engine.

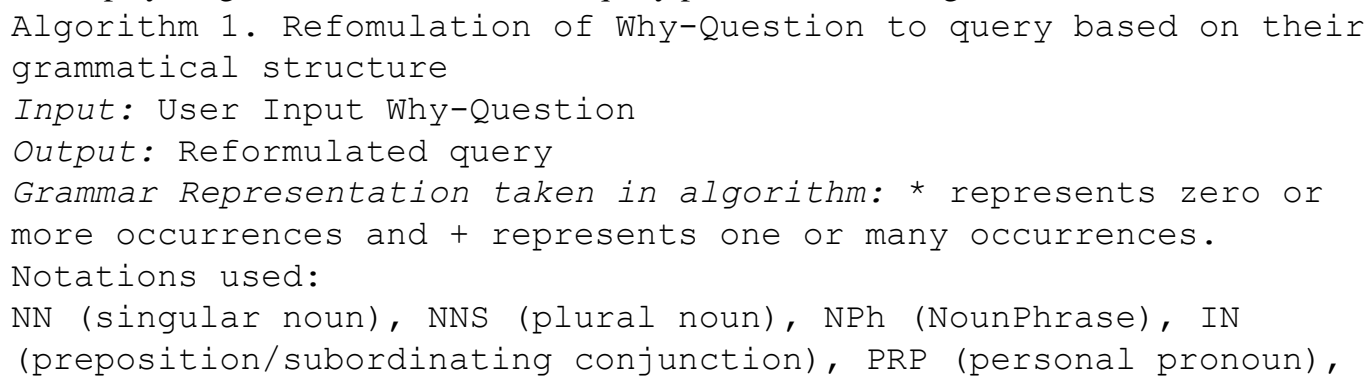


VBN (verb past principle), VBD (verb past tense), VB (verb), VBPh (verb phrase), VBP (verb, present tense not $3^{\text {rd }}$ person singular), VBZ (verb, present tense with $3^{\text {rd }}$ person singular), VBG (verb gerund), RB (adverb), RBR (comparative adverb), MD (modal), TO (infinite marker (to)), JJ (adjective), JJR (comparative adjective), CD (cardinal digit), WRB (wh-adverb), DT (determiner), CC (coordinating conjunction), I (or)

NN | PRP (NN)* | PRP NNS | NPh NPh $\rightarrow$ NPh NN | NN NPh | NPh IN

| MD VB | RB VBN VBPh $\rightarrow$ VBN IN | RB VBN | RB VBD

Procedure:

Step1: Find POS tagging of the question using NLTK

Step 2: Apply classification algorithm on the questions to find the type of questions

Step 3: If the type of why-question is Informational type:

Step 3a: Check if the pattern of question is Why VBP/VBZ NPh/NN $\left(\mathrm{TO}(\mathrm{VBPh}){ }^{*}(\mathrm{JJ})^{*}(\mathrm{IN}){ }^{*}(\mathrm{JJ})^{*}\right.$

$\mathrm{NPh})^{+}$, the reformulated query is $\{\mathrm{NPh}$ VBP|VB NPh|NN because $\}$ or $\{\mathrm{NPh}$ (IN JJ | JJ IN)

VBZ)

$(\mathrm{NPh})^{*}$ because \} (if no VBP/VB present in case of

Step 3b: Check if the pattern of question is Why NPh

$(\mathrm{VBZ}|\mathrm{VBG}| \mathrm{VBN}|\mathrm{VBPh}| \mathrm{VBP})^{+} \quad(\mathrm{IN}$

$\mathrm{NN}|\mathrm{NPh}| \mathrm{JJ} \mid \mathrm{CD}))^{*}$, the reformulated query is $\left\{(\mathrm{NPh})^{+}\right.$

$(\mathrm{VNP} \mid \mathrm{VBPh})^{*}(\mathrm{NPh}){ }^{*} \mathrm{IN} \mathrm{NPh}(\mathrm{IN} \mathrm{CD})$ *

because

Step 4: If the type of why-question is Historical type:

Step 4a: The pattern of question is Why $\operatorname{VBD} \operatorname{NPh}(\mathrm{RB})^{*} \operatorname{VBP}(\mathrm{NPh})$ * $(\mathrm{CD}|\mathrm{WRB}| \mathrm{TO} \mid \mathrm{IN})$ *

$(V B){ }^{*}(R B \mid I N) *(C D \mid N P h) *$ and the reformulated query is $\left\{\mathrm{NPh}(\mathrm{RB}){ }^{*} \operatorname{VBP}\right.$ (past tense) $\mathrm{NPh}$

$$
(\mathrm{RB}) \text { * }(\mathrm{IN}) \text { * }(\mathrm{CD})^{*}(\mathrm{NPh}) \text { * because }
$$

Step 5: If the type of why-question is Situational type:

Step 5a: If the pattern of question is Why (VBZ/VBP)* (NPh|NNS|NN) (JJ TO|RBR) *

$(\mathrm{VBZ}|\mathrm{VBN}| \mathrm{VBP})^{*}(\mathrm{IN} \mathrm{NPh} \mid \mathrm{NNP})^{+}$and VBP comprises one of $R B V B N \mid R B V B D$, the

$(\mathrm{VBN}|\mathrm{VBP}| \mathrm{VBD}) \quad(\mathrm{IN} \mathrm{NPh})^{+}$because

and requires user input regarding significance of

$\mathrm{RB}$.

Step 5b: If the pattern of question is Why NPh VBP TO NPh IN NPh IN $C D$, the reformulated

query is \{NPh VBP NPh IN NPh IN CD because\}

Step 5c: If the pattern of question is Why VBP VB VBP IN VBP, the reformulated query is

\{ VBP VB VBP IN VBP because\} 
Step 6: If the type of why-question is ComparativeSituational: Step 6a: If the pattern of question is WHY VBP NPh (VBP (NPh)* $(\mathrm{CC} \mid \mathrm{IN}) \quad(\mathrm{NPh}){ }^{*} \mathrm{VBP}$

$(\mathrm{CC} \mid \mathrm{IN}) \quad(\mathrm{NPh}){ }^{*} \mathrm{VBP}$

$\left.(\mathrm{JJ})^{*}\right)$, the reformulated query is $\{\mathrm{NPh}$ (VBP (NPh)*

$(J J)^{*}$ ) because $\}$ and if NPh is others, perform

anaphora resolution to find related

entity to it.

Step 6b: If the pattern of question is WHY VBZ (DT) * (NN|NPh)

$(\mathrm{VBD} \mid \mathrm{RBR})^{*}(\mathrm{CC} \mid \mathrm{IN})$

$(\mathrm{NPh})^{+}\left(\mathrm{CC} \mathrm{RB}\right.$ JJ IN NPh) ${ }^{*}$, the reformulated query

is $\{N N \mid N P h)(V B D \mid R B R)$ *

$$
(\mathrm{CC} \mid \mathrm{IN})(\mathrm{NPh})^{+}(\mathrm{CC} \mathrm{RB} \text { JJ IN NPh })^{*} \text { because }
$$

Step 6c: If the pattern of question is WHY VBP (NN|NPh) CC

$(\mathrm{NN} \mid \mathrm{NPh}) \quad(\mathrm{NNS}){ }^{*} \mathrm{VBP}$

$((R B) * J J)$, the reformulated query is $\{(N N \mid N P h) \quad C C$

$(N N|N P h| N N S)^{+}(R B)^{*} J J$

because

Step 6d: If the pattern of question is WHY (NPh VBP TO VBP) * NPh $(\mathrm{VBZ})^{*}$

(JJR $|R B R| J J) *$ IN (JJ $|N P h| N N \mid N N S)$, the reformulated query is $\{(\mathrm{VBP}$ TO $\mathrm{VBP})$ *

NPh (JJR|RBR/JJ) * IN (JJ|NPh|NN|NNS) because\} and

if the question begins

with (VBP TO VBP)* or ending with JJ, requires

user input to find the actual

need.

Step 7: If the type of why-question is Opinionated type:

Step 7a: If the pattern of question is Why NPh (VBD|VBZ) (JJ| NPh)

IN NPh, the

NPh because

reformulated query is $\{\mathrm{NPh}$ (VBD|VBZ) (JJ| NPh) IN

Step 7b: If the pattern of question is Why (VBZ| (VBD RB)) (DT)*

$\mathrm{NPh} \operatorname{VBP}(\mathrm{NPh}){ }^{*}$ IN NPh

$(\operatorname{VBD})^{*}(\mathrm{WRB} N \mathrm{NPh}){ }^{*} \operatorname{VBP}(\mathrm{NPh}){ }^{*}\left(\mathrm{JJR}_{\mathrm{IN}} \mathrm{DT} \mathrm{NPh}\right){ }^{*}$, the

reformulated query is

$\left\{\mathrm{NPh}(\mathrm{VBD} \mid \mathrm{VB}){ }^{*}(\mathrm{NPh})^{*} \mathrm{IN} \mathrm{NPh}(\mathrm{VBD} \mid \mathrm{VBP}){ }^{*}\right.$ because $\}$

Step 7c: If the pattern of question is Why (VBP/VBD) NPh (RB VBP) * $(\mathrm{VBPh} \mid \mathrm{VB})^{*}(\mathrm{NPh} \mid$

$\left.(\mathrm{TO} \mathrm{VB} J J)^{*} \mathrm{IN}(\mathrm{NPh} \mid \mathrm{VBPh}) \mid(\mathrm{RP} \mathrm{VBN})\right)^{*}$, the

reformulated query is $\{\mathrm{NPh}$ (VBPh)

$\mathrm{VBP} \mid \mathrm{VB})^{+}(\mathrm{NPh}|\mathrm{IN}| \mathrm{RP}|\mathrm{JJR}| \mathrm{TO}){ }^{*}(\mathrm{NPh} \mid \mathrm{VBPh}){ }^{*}$ because

The example of above algorithm is illustrated from Table 1 to Table 5.

\section{IMPLEMENTATION DETAILS}

This section describes implementation for user interface designed for question reformulation. The user interface provides an ease to user with functionalities of question. The implementation is performed using Tkinter module of Python and functionalities are performed using NLTK module of Python. 
Table 1. Example of Informational Why-Question and their query corresponding to their different patterns

\begin{tabular}{|c|c|c|c|}
\hline \multicolumn{4}{|c|}{ Informational Why-type Questions } \\
\hline \multicolumn{4}{|c|}{ Pattern 1: Why VBPIVBZ NPhINN (TO $(\mathrm{VBPh}) *(J J) *(I N) *(J J) *$ NPh $)+$} \\
\hline Question Example & Focus words & Focus terms & Query \\
\hline $\begin{array}{l}\text { Why are hush puppies called } \\
\text { hush puppies? }\end{array}$ & $\begin{array}{l}\text { Hush puppies called hush } \\
\text { puppies }\end{array}$ & $\mathrm{NPh} \mathrm{VBPh} \mathrm{NPh}$ & NPh VBP VBPh NPh because \\
\hline Why does sugar taste sweet? & $\begin{array}{l}\text { Sugar taste sweet or sugar } \\
\text { sweet }\end{array}$ & $\mathrm{NPh}$ VB NN & $\mathrm{NPh}$ VB (if VBZ) NN because \\
\hline $\begin{array}{l}\text { Why is cleanliness an } \\
\text { important requirement for } \\
\text { contact lenses? }\end{array}$ & $\begin{array}{l}\text { Cleanliness an important } \\
\text { requirement for contact } \\
\text { lenses }\end{array}$ & $\mathrm{NN} \mathrm{NPh} \mathrm{NPh}$ IN NPh & $\begin{array}{l}\text { In case of VBZ, if no VB, then } \\
\text { add IN } \\
\text { NN NPh NPh IN NPh because }\end{array}$ \\
\hline $\begin{array}{l}\text { Why is "fish" referred to as } \\
\text { "brain food"? }\end{array}$ & fish referred brain food & $\mathrm{NPh} \mathrm{VBPh} \mathrm{NPh}$ & $\mathrm{NPh}$ VBPh NPh because \\
\hline \multicolumn{4}{|c|}{ Pattern 2: Why NPh (VBZIVBGIVBN|VBPh|VBP)+ (IN NNINPh|JJ|CD))*: } \\
\hline $\begin{array}{l}\text { Why will cereal farmers } \\
\text { rejoice? }\end{array}$ & Cereal farmers rejoice & $\mathrm{NPh} \mathrm{VBPh}$ & $\mathrm{NPh}$ VBPh because \\
\hline $\begin{array}{l}\text { Why groups are } \\
\text { commutative? }\end{array}$ & Groups are commutative & NPh VBP JJ & $\mathrm{NPh}$ VBP JJ because \\
\hline $\begin{array}{l}\text { Why mathematics is } \\
\text { foundation for computer } \\
\text { science? }\end{array}$ & $\begin{array}{l}\text { Mathematics foundation } \\
\text { for computer science }\end{array}$ & $\mathrm{NPh} \mathrm{NPh}$ IN NPh & $\mathrm{NPh} \mathrm{NPh}$ IN NPh because \\
\hline $\begin{array}{l}\text { Why Lionel Messi known as } \\
\text { God of Football? }\end{array}$ & $\begin{array}{l}\text { Lionel Messi known God } \\
\text { of Football }\end{array}$ & $\begin{array}{l}\text { NPh VBPh NPh IN } \\
\text { NPh }\end{array}$ & $\mathrm{NPh}$ VBPh NPh IN NPh because \\
\hline
\end{tabular}

Table 2. Example of Historical Why-Question and their query corresponding to their pattern

\begin{tabular}{|c|c|c|c|}
\hline \multicolumn{4}{|l|}{ Historical Why-Questions } \\
\hline \multicolumn{4}{|c|}{ Pattern 1: Why VBD NPh $(\mathrm{RB}) *$ VBP $(\mathrm{NPh}) *(\mathrm{CD}|\mathrm{WRB}| \mathrm{TO} \mid \mathrm{IN}) *(\mathrm{VB}) *(\mathrm{RB} \mid \mathrm{IN}) *(\mathrm{CD} \mid \mathrm{NPh}) *$} \\
\hline $\begin{array}{l}\text { Why did the chicken cross } \\
\text { the road? }\end{array}$ & $\begin{array}{l}\text { The chicken crossed the } \\
\text { road }\end{array}$ & $\mathrm{NPh} \operatorname{VBP}$ (past tense) $\mathrm{NPh}$ & $\begin{array}{l}\text { NPh VBP (past tense) NPh } \\
\text { because }\end{array}$ \\
\hline $\begin{array}{l}\text { Why was Pearl Harbor } \\
\text { bombed? }\end{array}$ & Pearl Harbor bombed & NPh VBP (past tense) & $\begin{array}{l}\text { NPh VBP (past tense) } \\
\text { because }\end{array}$ \\
\hline $\begin{array}{l}\text { Why United States entered } \\
\text { World War } 2 \text { ? }\end{array}$ & $\begin{array}{l}\text { United States entered World } \\
\text { War } 2\end{array}$ & $\mathrm{NPh}$ VBP (past tense) NPh & $\begin{array}{l}\text { NPh VBP (past tense) NPh } \\
\text { because }\end{array}$ \\
\hline $\begin{array}{l}\text { Why was Paris given board } \\
\text { boulevards after } 1848 ?\end{array}$ & $\begin{array}{l}\text { Paris given board } \\
\text { boulevards after } 1848\end{array}$ & $\mathrm{NPh}$ VBP NPh IN CD & $\begin{array}{l}\text { If CD- add In conjunction- } \\
\text { plays significance } \\
\text { NPh VBP NPh IN CD } \\
\text { because }\end{array}$ \\
\hline $\begin{array}{l}\text { Why did india not win the } \\
\text { women's cricket t } 20 \text { world } \\
\text { cup in } 2020 \text { ? }\end{array}$ & $\begin{array}{l}\text { India not win the women's } \\
\text { cricket t } 20 \text { world cup in } \\
2020\end{array}$ & $\mathrm{NPh}(\mathrm{RB}$ VBP) NPh IN CD & $\begin{array}{l}\text { If CD- add In conjunction- } \\
\text { plays significance } \\
\text { NPh (RB VBP) NPh IN } \\
\text { CD because }\end{array}$ \\
\hline
\end{tabular}


Table 3. Example of Situational Why-Question and their query corresponding to their patterns

\begin{tabular}{|c|c|c|c|}
\hline \multicolumn{4}{|c|}{ Situational Why-type Questions } \\
\hline \multicolumn{4}{|c|}{ Pattern 1: Why (VBZIVBP)* (NPhINNSINN) (JJ TOIRBR)*(VBZIVBNIVBP)* (IN NPh I NNP)+ } \\
\hline $\begin{array}{l}\text { Why India is still come } \\
\text { under the category of } \\
\text { developing country? }\end{array}$ & $\begin{array}{l}\text { India come under the } \\
\text { category of developing } \\
\text { country }\end{array}$ & $\begin{array}{l}\text { NPh VBN IN NPh IN } \\
\text { NPh }\end{array}$ & $\begin{array}{l}\text { NPh VBN IN NPh IN NPh } \\
\text { because }\end{array}$ \\
\hline $\begin{array}{l}\text { Why is street food risky to } \\
\text { eat in india during monsoon? }\end{array}$ & $\begin{array}{l}\text { Street food risky to eat in } \\
\text { India during monsoon }\end{array}$ & $\begin{array}{l}\text { NPh JJ TO VBP IN } \\
\text { NPh IN NPh }\end{array}$ & $\begin{array}{l}\text { NPh JJ TO VBP IN NPh IN NPh } \\
\text { because }\end{array}$ \\
\hline $\begin{array}{l}\text { Why does temperature } \\
\text { change during the seasons? }\end{array}$ & $\begin{array}{l}\text { Temperature change } \\
\text { during the seasons }\end{array}$ & NN VBP IN NPh & NPh VBN IN NPh because \\
\hline \multicolumn{4}{|c|}{ Pattern 2: Why VBP VB VBP IN VBP } \\
\hline $\begin{array}{l}\text { Why should chicken be well } \\
\text { cooked before eating? }\end{array}$ & $\begin{array}{l}\text { Chicken be well cooked } \\
\text { before eating }\end{array}$ & VBP VB VBP IN VBP & VBP VB VBP IN VBP because \\
\hline
\end{tabular}

Table 4. Example of ComparativeSituational Why-Question and their query corresponding to their patterns

\begin{tabular}{|c|c|c|c|}
\hline \multicolumn{4}{|c|}{ ComparativeSituational Why Questions } \\
\hline \multicolumn{4}{|c|}{ Pattern 1: WHY VBP NPh (VBP (NPh)* (CCIIN) (NPh)* VBP (JJ)*) } \\
\hline $\begin{array}{l}\text { Why do stock prices rise } \\
\text { and fall? }\end{array}$ & Stock prices rise and fall & NPh VBP CC VBP & $\mathrm{NPh}$ VBP CC VBP because \\
\hline $\begin{array}{l}\text { Why do some chickens lay } \\
\text { brown eggs while others lay } \\
\text { white? }\end{array}$ & $\begin{array}{l}\text { Some chickens lay brown } \\
\text { eggs while others lay } \\
\text { white }\end{array}$ & $\begin{array}{l}\text { NPh VBP NPh IN NPh } \\
\text { VBP JJ }\end{array}$ & $\begin{array}{l}\text { NPh VBP NPh IN NPh VBP JJ } \\
\text { because }\end{array}$ \\
\hline \multicolumn{4}{|c|}{ Pattern 2: WHY VBZ (DT)* (NNINPh) $($ VBDIRBR)* (CCIIN) $($ NPh $)+($ CC RB JJ IN NPh)* } \\
\hline $\begin{array}{l}\text { Why is the sky red at sunset } \\
\text { and also colorful at sunrise? }\end{array}$ & $\begin{array}{l}\text { Sky red at sunset and } \\
\text { colourful at sunrise }\end{array}$ & $\begin{array}{l}\text { VBD IN NPh CC JJ } \\
\text { IN NPh }\end{array}$ & $\begin{array}{l}\text { VBD IN NPh CC JJ IN NPh } \\
\text { because }\end{array}$ \\
\hline $\begin{array}{l}\text { Why does a seashell sound } \\
\text { like the ocean? }\end{array}$ & $\begin{array}{l}\text { a seashell sound like the } \\
\text { ocean }\end{array}$ & $\mathrm{NPh}$ NN IN NPh & NPh NN IN NPh because \\
\hline $\begin{array}{l}\text { Why is mind and muscle } \\
\text { memory important for } \\
\text { exercise enthusiasts? }\end{array}$ & $\begin{array}{l}\text { Mind and muscle } \\
\text { memory important for } \\
\text { exercise enthusiasts }\end{array}$ & $\begin{array}{l}\text { NPh CC NPh JJ IN } \\
\text { NPh }\end{array}$ & $\mathrm{NPh}$ CC NPh JJ IN NPh because \\
\hline $\begin{array}{l}\text { Why is obesity higher in } \\
\text { developing and developed } \\
\text { countries? }\end{array}$ & $\begin{array}{l}\text { Obesity higher in } \\
\text { developing and developed } \\
\text { countries }\end{array}$ & NN RBR IN NPH & NN RBR IN NPH because \\
\hline \multicolumn{4}{|c|}{ Pattern 3: WHY VBP (NNINPh) CC (NNINPh) (NNS)* VBP ((RB)* JJ) } \\
\hline $\begin{array}{l}\text { Why do movie and TV stars } \\
\text { get paid so much? }\end{array}$ & $\begin{array}{l}\text { Movie and TV stars get } \\
\text { paid so much }\end{array}$ & $\begin{array}{l}\text { NN CC NN NNS VBP } \\
\text { RB JJ }\end{array}$ & $\begin{array}{l}\text { NN CC NN NNS VBP RB JJ } \\
\text { because }\end{array}$ \\
\hline $\begin{array}{l}\text { Why Zomato and Swiggy } \\
\text { are getting famous? }\end{array}$ & $\begin{array}{l}\text { Zomato and Swiggy } \\
\text { getting famous }\end{array}$ & $\mathrm{NPh}$ CC NPh VBP JJ & $\mathrm{NPh}$ CC NPh VBP JJ because \\
\hline \multicolumn{4}{|c|}{ Pattern 4: WHY (NPh VBP TO VBP)* NPh (VBZ)* (JJR|RBR|JJ)* IN (JJINPh|NNINNS) } \\
\hline $\begin{array}{l}\text { Why light travels faster than } \\
\text { sound? }\end{array}$ & $\begin{array}{l}\text { Light travels faster than } \\
\text { sound }\end{array}$ & NPh RBR IN NN & NPh RBR IN NN because \\
\hline $\begin{array}{l}\text { Why Instagram is popular } \\
\text { than Facebook nowadays? }\end{array}$ & $\begin{array}{l}\text { Instagram popular than } \\
\text { Facebook nowadays }\end{array}$ & NPh JJ IN NPh NNS & NPh JJ IN NPh NNS because \\
\hline $\begin{array}{l}\text { Why tourists prefer to visit } \\
\text { Niagara Falls than Venice } \\
\text { Beach? }\end{array}$ & $\begin{array}{l}\text { Tourists prefer to visit } \\
\text { Niagara Falls than Venice } \\
\text { Beach }\end{array}$ & $\begin{array}{l}\text { NPh VBP TO VBP } \\
\text { NPh IN NPh }\end{array}$ & $\begin{array}{l}\text { NPh VBP TO VBP NPh IN NPh } \\
\text { because }\end{array}$ \\
\hline
\end{tabular}


Table 5. Example of Opinionated Why-Question and their query corresponding to their patterns

\begin{tabular}{|c|c|c|c|}
\hline \multicolumn{4}{|c|}{ Opinionated Why-type Questions } \\
\hline \multicolumn{4}{|c|}{ Pattern 1: Why NPh (VBD|VBZ) (JJ/ NPh) IN NPh } \\
\hline $\begin{array}{l}\text { Why B.B. King named his } \\
\text { guitar as "Lucille"? }\end{array}$ & $\begin{array}{l}\text { B.B. King named his } \\
\text { guitar as "Lucille" }\end{array}$ & NPh VBD NPh IN NPh & NPh VBD NPh IN NPh because \\
\hline $\begin{array}{l}\text { Why internet is important } \\
\text { for your life? }\end{array}$ & $\begin{array}{l}\text { Internet important for } \\
\text { your life }\end{array}$ & NPh JJ IN NPh & NPh JJ IN NPh because \\
\hline \multicolumn{4}{|c|}{$\begin{array}{l}\text { Pattern 2: Why (VBZI(VBD RB)) (DT)* NPh VBP (NPh)* IN NPh (VBD)*(WRB NPh)* VBP (NPh)* (JJR IN } \\
\text { DT NPh)* }\end{array}$} \\
\hline $\begin{array}{l}\text { Why didn't Socrates } \\
\text { leave Athens after he was } \\
\text { convicted? }\end{array}$ & $\begin{array}{l}\text { Socrates didn't leave } \\
\text { Athens after he was } \\
\text { convicted }\end{array}$ & $\begin{array}{l}\text { NPh VBD VB NPh IN } \\
\text { NPh VBD VBP }\end{array}$ & $\begin{array}{l}\text { NPh VBD VB NPh IN NPh } \\
\text { VBD VBP because }\end{array}$ \\
\hline $\begin{array}{l}\text { Why is the gastrointestinal } \\
\text { tract of animals that eat } \\
\text { grass longer than a humans? }\end{array}$ & $\begin{array}{l}\text { Gastrointestinal tract of } \\
\text { animals that eat grass } \\
\text { longer than a humans }\end{array}$ & $\begin{array}{l}\text { (NPh) IN NPh WDT } \\
\text { VBP NPh JJR IN NPh }\end{array}$ & $\begin{array}{l}\text { (NPh) IN NPh WDT VBP NPh } \\
\text { JJR IN NPh }\end{array}$ \\
\hline
\end{tabular}

The reformulation interface opens with a welcome page indicating 'Why-Question to Query Reformulator'. Next page has an input label which asks user to input Why-question. The input question is processed in the next window to calculate its functionalities which are : Classification into its type and answer type, Named entity recognition, POS tagging, Tokenization, constituency parsing, dependency parsing with tree formation, Sentiment analysis, lemmatization, noun phrase extraction; the details of which are discussed in Section 4. The functionality button is clicked by the user and its result is displayed on the Python console.

Next window consists of the button for 'question to query' which reformulates input why-question to query according to an algorithm discussed in Section 5. The output query is posed on the Google search engine to extract relevant web pages to it for which user feedback is taken. The performance of user feedback is discussed in Section 7 below.

The snapshots of the user interface developed are illustrated below:

\section{RESULTS AND DISCUSSION}

A user interface is implemented which takes input why-question from user and applies various functionalities to compute the value of various components such as sub-classification and determining its type and answer type, named entities recognition, tokenization, POS tagging, constituency and dependency parsing, noun phrase extraction. After determining its type and functionalities, patterns of why-type questions are analyzed. For each pattern, rules are formulated which finds the important terms from question and helps to formulate query that can be posed on search engine to extract relevant web pages. The web pages returned are rated by the 10 users of different age groups on the scale of 1 to 10 which measures how many pages out of 10 returned pages are relevant to users.

10 questions of each question type are reformulated and posed on Google search engine for a set of web pages. Returned web pages for each question are distributed to 10 users of different age group to take their feedback on the relevancy of the returned web pages. Further in order to calculate precision of an algorithm, a group technique is adopted where 10 users collaborate together as a team and arrive at a scoring of pages returned for each question type. This further helps to calculate the final precision which finds the ratio of total number of relevant documents retrieved by the total number of documents retrieved (“Precision and Recall”, n.d.) 


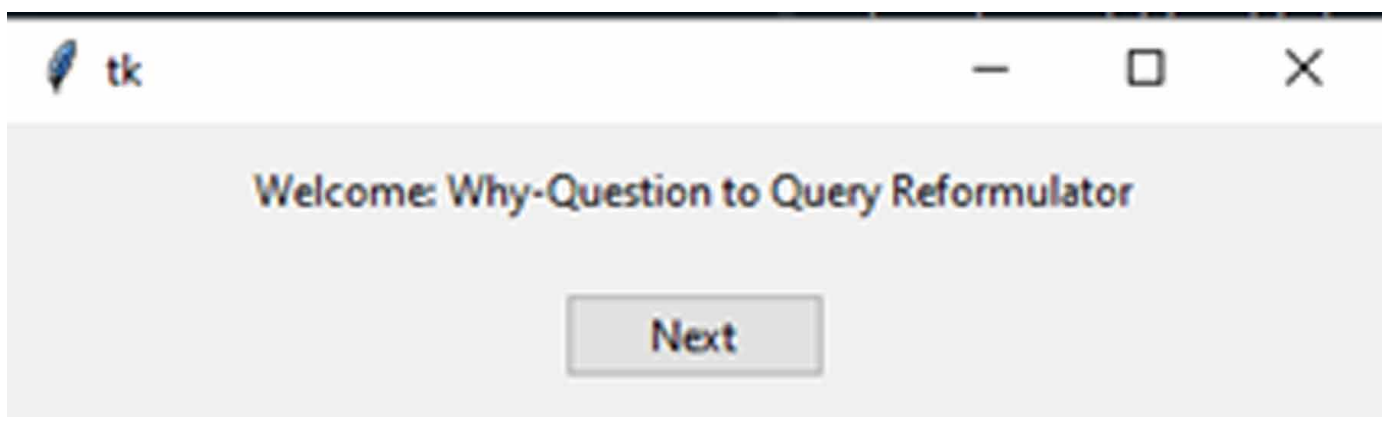

Figure 2. Asking user to input why-question and button to compute its functionalities

\section{User input for Question

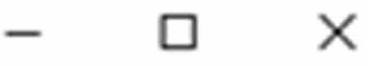

Enter a why-question

Why sun rises in east?

Submit

\section{Functionalities on user's question}

Figure 3. Buttons for calculating different components of question

Components of question

$-\square \times$

Classification to know question and answer type

Named entity

POS Tagging

Tokens of question

Constituency parsing

Dependency parsing

Sentiment Analysis

Lematication

Noun Phrases

Reformulated Query 


\section{Reformulating Questio...}
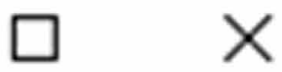

\section{Focus terms}

\section{Reformulated Query}

\section{Search on Google}

\section{User Feedback}

Figure 5. Scaler to take input from user regarding satisfaction of retrieved Google results based on reformulated query

User Feedback on valid...
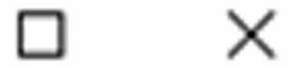

\section{How relevant are web pages?}

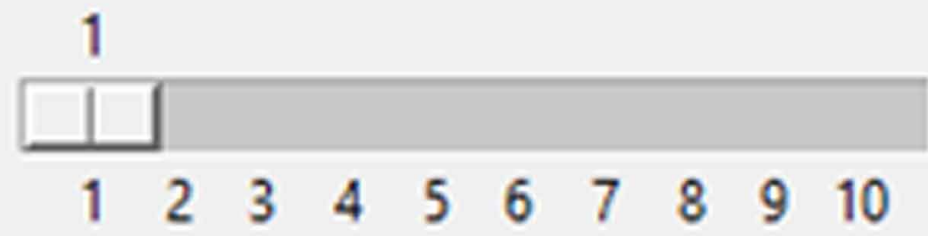


Figure 6. Output of different functionalities applied on sample user input question 'Why sun rises in east?'

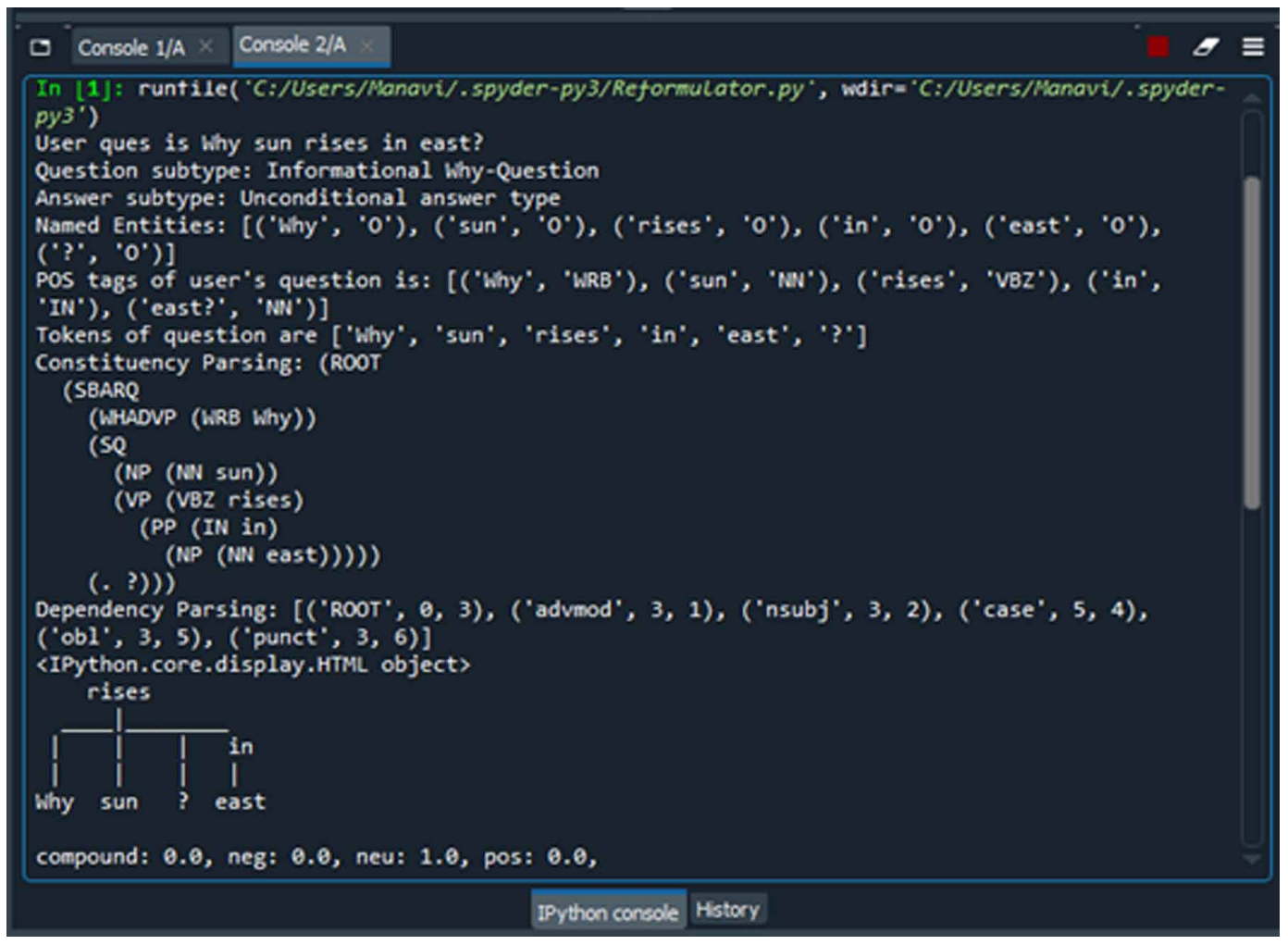

Figure 7. Output of lemmatization, focus, noun phrases and reformulated query to the input user question 'Why sun rises in east?'

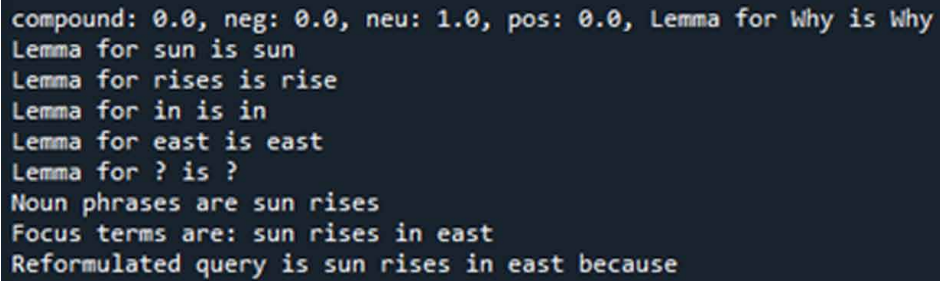


Figure 8. Google web pages corresponding to the reformulated query 'sun rises in east because'

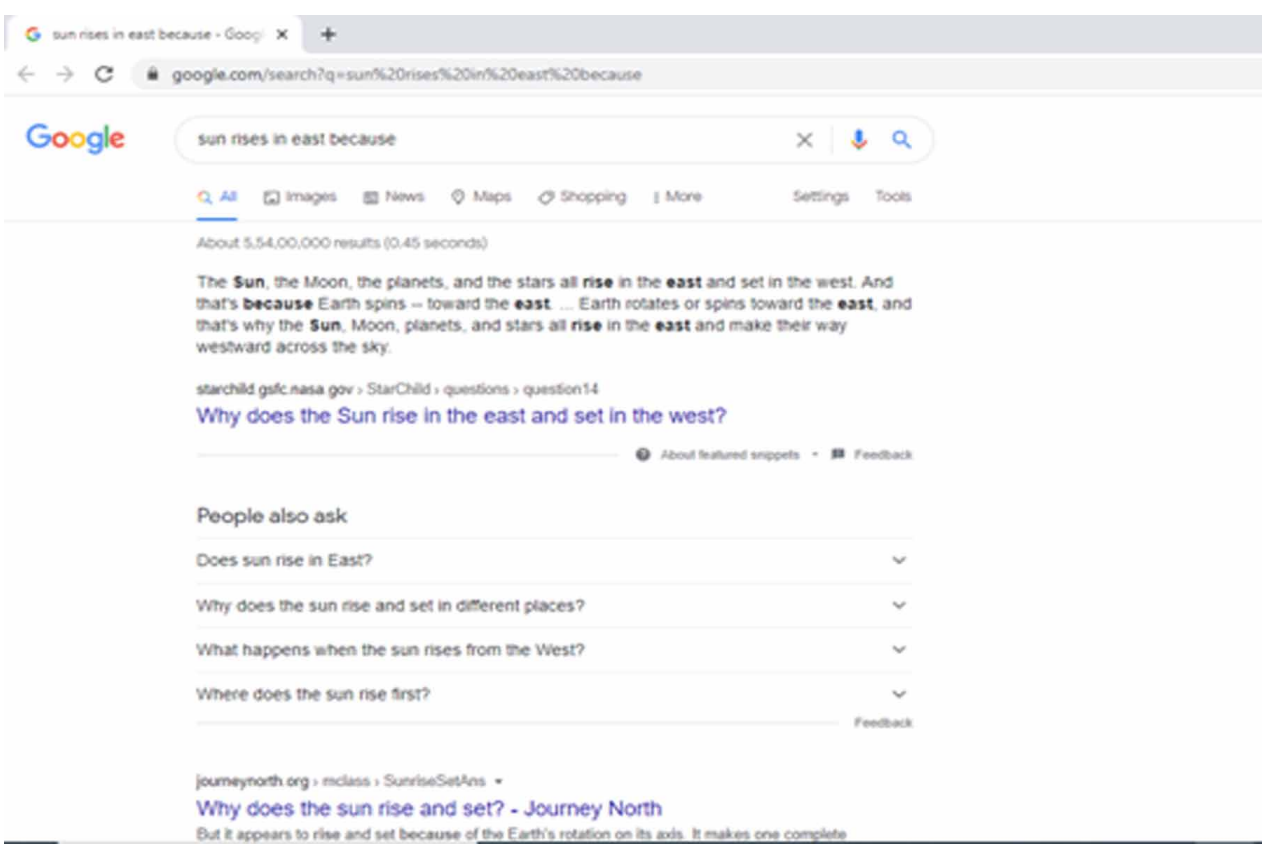

Figure 9. Google web pages corresponding to the reformulated query 'sun rises in east because' contd.

$G$ sun rises in east because - Goog $\times+$
$\leftarrow \rightarrow C$ google.com/search?q=sun\%20rises\%20in\%20east\%20because
Google $\quad$ sun rises in east because

brainly in > Physics , Secondary School .

why does sun rise in east and set in the west? - Brainly.in

Sep 12, 2018 - Eut it appears to rise and set because of the Earth's rotation on its axis. It

makes one complete turn every 24 hours. It turns toward the east

2 answers

wwwtoppr com , ask , question > on-the-earth-the-sun...

On the Earth, the sun rises in the east and sets in the west.Will ...

The sun rises in the east and sets in the west same on venus, Because the planet is having a retrograde rotation. Unike other planets in the solar system that ...

1 answer

wwwnaturalnavigator.com , find-your-way-using , sun *

How to navigate using the Sun - The Natural Navigator

The sun rises due east on only two days of the year, the equinoxes, near... This is important

because this is the moment that the sun has stopped rising, ie. it is ...

\section{Searches related to sun rises in east because}

sun rises in the east and sets in the west

the sun rises in the

the sun rises in the east correct sentence

sun rises in which direction
Why sun rises in the east in hindi

sun rises in which direction in india

where does the sun rise first

how sun moves from east to west 


\section{User Feedback on valid... - $\quad$ - $\times$}

\section{How relevant are web pages?}

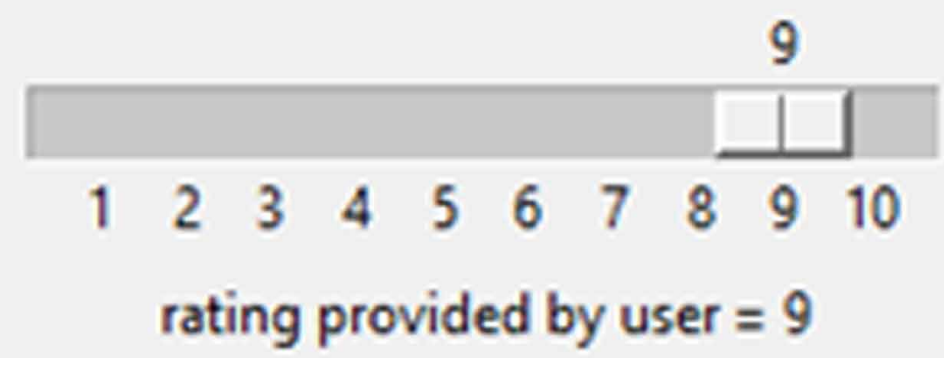

Figure 11. Precision of users feedback based on different Why-type questions

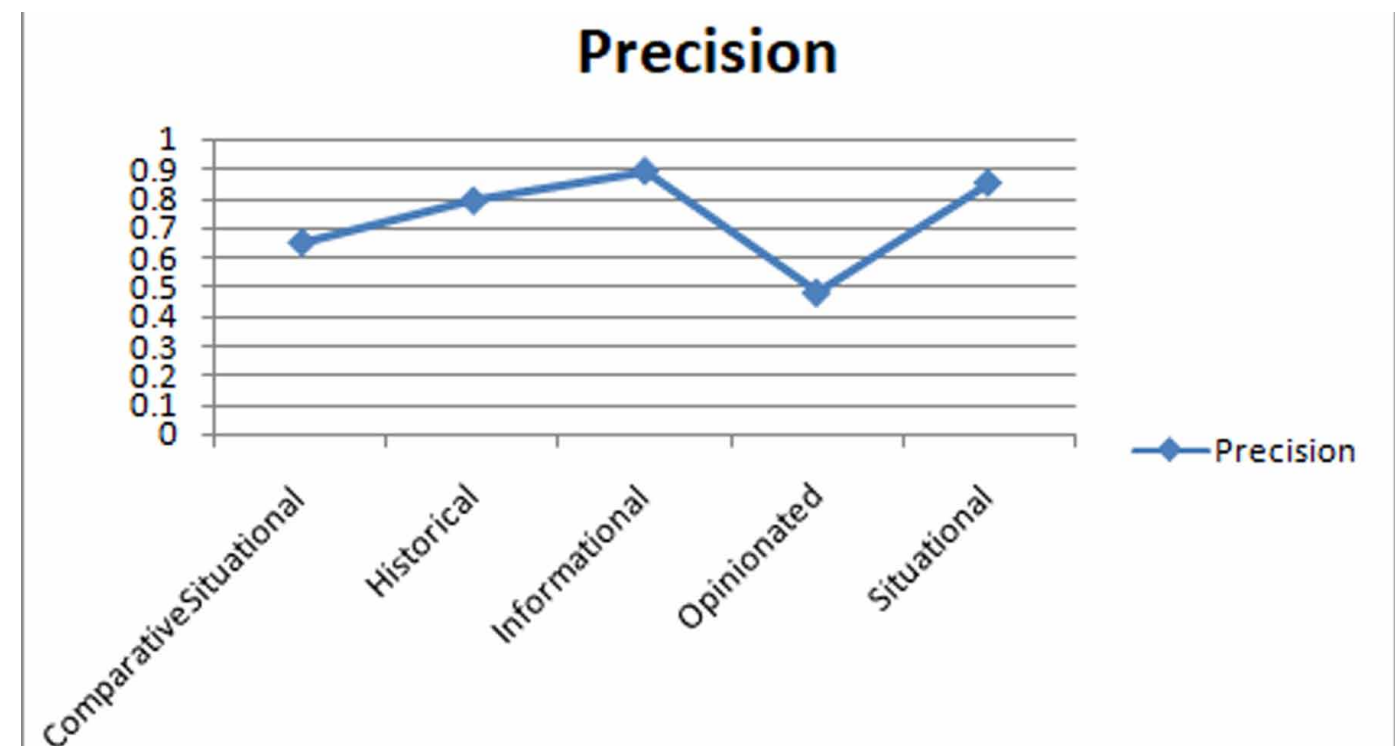

Precision $=\frac{\text { Totalnumber of relevant documents retrieved by search }}{\text { Totalnumber of documents retrieved by search }}$

Table 6, briefs the precision achieved for each Why-question type which is illustrated further in Figure 11.

The results of precision clearly depict that informational why-type questions are best answered by Google search. In both ComparativeSituational and situational questions, input is required for some type of questions which needs further clarification on the part of understanding the need of user. In Historical type questions, most of the questions can be appropriately answered but some questions 
Table 6. Precision achieved for each Why-question type

\begin{tabular}{|l|l|}
\hline QuestionType & Precision \\
\hline ComparativeSituational & 0.65 \\
\hline Historical & 0.79 \\
\hline Informational & 0.89 \\
\hline Opinionated & 0.48 \\
\hline Situational & 0.85 \\
\hline
\end{tabular}

which seek answering with respect to the particular time period suffers with inappropriate answers. The case of opinionated why-question shows very drastic results because answering to such questions differ with person's opinion and thus can't be answered properly if directly posed on search engine. Thus, the results very well infer that reformulation alone can't improve the accuracy of why-question answering but interaction of user is also required in many questions which can clarify their actual demand in the answering.

\section{COMPARISON OF OUR PROPOSED APPROACH WITH OTHER WORK ON QUESTION REFORMULATION}

Table 7 discusses the work on question reformulation with our proposed approach of question reformulation on Why-type QAS. 
Table 7. Comparison of our proposed approach with other work of question reformulation on QAS

\begin{tabular}{|c|c|c|c|}
\hline References & $\begin{array}{l}\text { Methodology for } \\
\text { reformulation }\end{array}$ & Question type & Performance \\
\hline Umamehaswari et al. (2012) & $\begin{array}{l}\text { Semantic based technique, } \\
\text { patterns based on lexical, } \\
\text { syntactic and semantic } \\
\text { constraints are generated }\end{array}$ & $\begin{array}{l}\text { Who, what, where, when, how, } \\
\text { which }\end{array}$ & $\begin{array}{l}0.498 \text { precision with candidate } \\
\text { answer, } 0.588 \text { with generated } \\
\text { patterns }\end{array}$ \\
\hline Iturbe Herrera et al. (2021) & $\begin{array}{l}\text { Different grammatical elements } \\
\text { identified on question classes to } \\
\text { locate question focus, CNN was } \\
\text { trained on dataset }\end{array}$ & $\begin{array}{l}\text { TREC V2, WebQues dataset } \\
\text { involving what, when, who, } \\
\text { where question type }\end{array}$ & $\begin{array}{l}\text { 96.84\% accuracy with TREC } \\
\text { dataset, } 90.91 \% \text { on WebQues, } \\
87.37 \% \text { on WikiMovies, } 82.5 \% \\
\text { on TREc } 10 \text { and } 96.63 \% \text { on } \\
\text { SimpleQues dataset }\end{array}$ \\
\hline Esposito et al.(2021) & $\begin{array}{l}\text { Query expansion approach } \\
\text { comprising } 4 \text { steps viz. } \\
\text { Question processing } \\
\text { and expandable terms } \\
\text { identification, candidate } \\
\text { expansion terms extraction and } \\
\text { contextualization, candidate } \\
\text { expansion terms ranking and } \\
\text { filtering and query formulation }\end{array}$ & $\begin{array}{l}\text { Person, Entity, Location, } \\
\text { Date, Description as top level } \\
\text { categories, and Address, City, } \\
\text { Region, Artifact as bottom level } \\
\text { categories }\end{array}$ & $\begin{array}{l}\text { Improvement in accuracy by } \\
9.1 \% \text { over the approaches } \\
\text { based on MultiWordNet, to } \\
15.7 \% \text { over Word } 2 \text { vec model } \\
\text { and } 37.1 \% \text { over an approach } \\
\text { without QE. }\end{array}$ \\
\hline Vakulenko et al. (2021) & $\begin{array}{l}\text { Question Rewriting (QR) } \\
\text { as a component of QA task. } \\
\text { Different question rewriting } \\
\text { (QR) models were proposed } \\
\text { which boosts the performance } \\
\text { of answer extraction }\end{array}$ & $\begin{array}{l}\text { Conversational question } \\
\text { answering }\end{array}$ & $\begin{array}{l}\text { Achieved maximum } 0.81 \\
\text { ROUGE score on Transformer } \\
++ \text { QR model in comparison } \\
\text { to } 0.84 \text { human performance on } \\
\text { CANARD test set with } 0.90 \\
\text { ROUGE score on TREC CAsT } \\
\text { test set in comparison to } 1.00 \\
\text { human performance. }\end{array}$ \\
\hline Verberne (2010) & $\begin{array}{l}\text { No reformulation concept was } \\
\text { introduced }\end{array}$ & Why-type question answering & $\begin{array}{l}\text { Success@ } 150 \text { is } 78.5 \% \text {, for } \\
21.5 \% \text { of questions, there was } \\
\text { no answer retrieved in top- } 150 \\
\text { documents retrieved }\end{array}$ \\
\hline Proposed Work & $\begin{array}{l}\text { Categorized Why-type } \\
\text { questions, identified different } \\
\text { patterns corresponding to each } \\
\text { answer type, reformulates them } \\
\text { into a query based on algorithm }\end{array}$ & Why-type question answering & $\begin{array}{l}\text { Maximum precision of } 0.89 \\
\text { for Informational Why-type } \\
\text { questions }\end{array}$ \\
\hline
\end{tabular}

\section{CONCLUSION}

The paper performs reformulation of why-type questions to query depending on different classes of why-type questions according to taxonomy proposed by the research in Breja and Jain (2018). With an algorithm, a user interface is also designed which asks user to input why-question, calculates different components of question, reformulates it into a query, and finally takes feedback on the web pages retrieved by a reformulated query. The method achieves precision of $89 \%$ in Informational-type and $48 \%$ in Opinionated-type why-questions. In future, performance of opinionated questions will be improved by incorporating interactive query refinement techniques where system will interact with user and take input at subsequent steps which will help the system to understand properly the need of such questions. 


\section{REFERENCES}

Bengfort, B. (2018, June 22). Syntax Parsing with CoreNLP and NLTK. bbengfort.github.io

Bernhard, D. (2010, August). Query expansion based on pseudo relevance feedback from definition clusters. In Coling 2010 (pp. 54-62). Posters.

Boldi, P., Bonchi, F., Castillo, C., \& Vigna, S. (2011). Query reformulation mining: Models, patterns, and applications. Information Retrieval, 14(3), 257-289. doi:10.1007/s10791-010-9155-3

Breja, M., \& Jain, S. K. (2017). Why-type Question Classification in Question Answering System. In FIRE (pp. 149-153). Working Notes.

Breja, M., \& Jain, S. K. (2018, September). Analysis of Why-Type Questions for the Question Answering System. In European Conference on Advances in Databases and Information Systems (pp. 265-273). Springer. doi:10.1007/978-3-030-00063-9_25

Esposito, M., Damiano, E., Minutolo, A., De Pietro, G., \& Fujita, H. (2020). Hybrid query expansion using lexical resources and word embeddings for sentence retrieval in question answering. Information Sciences, 514 , 88-105. doi:10.1016/j.ins.2019.12.002

Habernal, I., Konopík, M., \& Rohlík, O. (2012). Question Answering. In Next Generation Search Engines: Advanced Models for Information Retrieval (pp. 304-343). IGI Global. doi:10.4018/978-1-4666-0330-1.ch014

Herdagdelen, A., Ciaramita, M., Mahler, D., Holmqvist, M., Hall, K., Riezler, S., \& Alfonseca, E. (2010, July). Generalized syntactic and semantic models of query reformulation. In Proceedings of the 33rd international ACM SIGIR conference on Research and development in information retrieval (pp. 283-290). doi: $10.1145 / 1835449.1835498$

Iturbe Herrera, A., Castro Sánchez, N. A., \& Mújica Vargas, D. (2021). Rule-Based Spanish Multiple Question Reformulation and their Classification using a Convolutional Neuronal Network. Computación y Sistemas, 25(1). Advance online publication. doi:10.13053/cys-25-1-3895

Kangavari, M. R., Ghandchi, S., \& Golpour, M. (2008). Information retrieval: Improving question answering systems by query reformulation and answer validation. World Academy of Science, Engineering and Technology, $48,303-310$.

Kosseim, L., \& Yousefi, J. (2008). Improving the performance of question answering with semantically equivalent answer patterns. Data \& Knowledge Engineering, 66(1), 63-67. doi:10.1016/j.datak.2007.07.010

Levengood, C. (2020, January 6). Named Entity Recognition in Python with Stanford-NER and Spacy. https:// lvngd.com/blog/named-entity-recognition-in-python-with-stanford-ner-and-spacy/

Liu, Y. H., \& Belkin, N. J. (2008, October). Query reformulation, search performance, and term suggestion devices in question-answering tasks. In Proceedings of the second international symposium on Information interaction in context (pp. 21-26). doi:10.1145/1414694.1414702

Moldovan, D., Pasca, M., Harabagiu, S., \& Surdenau, M. (2003). Performance Issues and Error Analysis in an Open-Domain Question Answering System. ACM Transactions on Information Systems, 21(2), 133-154. doi:10.1145/763693.763694

Molla, D. (2009). From Minimal Logical Forms for Answer Extraction to Logical Graphs for Question Answering. Searching Answers: Festschrift in Honour of Michael Hess on the Occasion of His 60th Birthday. MV-Wissenschaft.

Musa, R., Wang, X., Fokoue, A., Mattei, N., Chang, M., Kapanipathi, P., \& Witbrock, M. (2018, November). Answering science exam questions using query reformulation with background knowledge. In Automated Knowledge Base Construction. AKBC.

Ooi, J., Ma, X., Qin, H., \& Liew, S. C. (2015, August). A survey of query expansion, query suggestion and query refinement techniques. In 2015 4th International Conference on Software Engineering and Computer Systems (pp. 112-117). IEEE. doi:10.1109/ICSECS.2015.7333094 
Pires, R. M. P. (2012). Query classification and expansion in just. Ask question answering system [Doctoral dissertation]. Instituto Superior Tcnico.

Prabhakaran S. (n.d.). Lemmatization Approaches with Examples in Python. https://www.machinelearningplus. com/nlp/lemmatization-examples-python/

Precision and recall. (n.d.). Retrieved July 09, 2021, from https://en.wikipedia.org/wiki/Precision_and_recall

Soricut, R., \& Brill, E. (2006). Automatic question answering using the web: Beyond the Factoid. Information Retrieval, 9(2), 191-206. doi:10.1007/s10791-006-7149-y

Umamehaswari, M., Ramprasath, M., \& Hariharan, S. (2012, December). Improved question answering system by semantic refomulation. In 2012 Fourth International Conference on Advanced Computing (ICoAC) (pp. 1-4). IEEE. doi:10.1109/ICoAC.2012.6416824

Vakulenko, S., Longpre, S., Tu, Z., \& Anantha, R. (2021, March). Question rewriting for conversational question answering. In Proceedings of the 14th ACM International Conference on Web Search and Data Mining (pp. 355-363). doi:10.1145/3437963.3441748

Verberne, S. (2010). Search of the Why: Developing a system for answering why-questions. Academic Press.

Manvi Breja is a PhD Scholar from National Institute of Technology, Kurukshetra. She has completed her M. Tech from YMCA University, Faridabad, India. Her area of interest includes Information Retrieval, Data Mining and Natural Language Processing.

Sanjay Kumar Jain, PhD (MNNIT, Allahabad, India), is a Professor in the Department of Computer Engineering at NIT Kurukshetra, India. He is involved in research and has 30 year experience of teaching. His current research areas include database, data mining, information retrieval, and requirements engineering. 